\title{
Sonine Transform Associated to the Dunkl Kernel on the Real Line ${ }^{\star}$
}

Fethi SOLTANI

Department of Mathematics, Faculty of Sciences of Tunis, Tunis-El Manar University, 2092 Tunis, Tunisia

E-mail: Fethi.Soltani@fst.rnu.tn

Received June 19, 2008, in final form December 19, 2008; Published online December 26, 2008

Original article is available at http://www.emis.de/journals/SIGMA/2008/092/

\begin{abstract}
We consider the Dunkl intertwining operator $V_{\alpha}$ and its dual ${ }^{t} V_{\alpha}$, we define and study the Dunkl Sonine operator and its dual on $\mathbb{R}$. Next, we introduce complex powers of the Dunkl Laplacian $\Delta_{\alpha}$ and establish inversion formulas for the Dunkl Sonine operator $S_{\alpha, \beta}$ and its dual ${ }^{t} S_{\alpha, \beta}$. Also, we give a Plancherel formula for the operator ${ }^{t} S_{\alpha, \beta}$.
\end{abstract}

Key words: Dunkl intertwining operator; Dunkl transform; Dunkl Sonine transform; complex powers of the Dunkl Laplacian

2000 Mathematics Subject Classification: 43A62; 43A15; 43A32

\section{Introduction}

In this paper, we consider the Dunkl operator $\Lambda_{\alpha}, \alpha>-1 / 2$, associated with the reflection group $\mathbb{Z}_{2}$ on $\mathbb{R}$. The operators were in general dimension introduced by Dunkl in [2] in connection with a generalization of the classical theory of spherical harmonics; they play a major role in various fields of mathematics $[3,4,5]$ and also in physical applications [6].

The Dunkl analysis with respect to $\alpha \geq-1 / 2$ concerns the Dunkl operator $\Lambda_{\alpha}$, the Dunkl transform $\mathcal{F}_{\alpha}$ and the Dunkl convolution $*_{\alpha}$ on $\mathbb{R}$. In the limit case $(\alpha=-1 / 2) ; \Lambda_{\alpha}, \mathcal{F}_{\alpha}$ and $*_{\alpha}$ agree with the operator $d / d x$, the Fourier transform and the standard convolution respectively.

First, we study the Dunkl Sonine operator $S_{\alpha, \beta}, \beta>\alpha$ :

$$
S_{\alpha, \beta}(f)(x):=\frac{\Gamma(\beta+1)}{\Gamma(\beta-\alpha) \Gamma(\alpha+1)} \int_{-1}^{1} f(x t)\left(1-t^{2}\right)^{\beta-\alpha-1}(1+t)|t|^{2 \alpha+1} d t
$$

and its dual ${ }^{t} S_{\alpha, \beta}$ connected with these operators. Next, we establish for them the same results as those given in $[8,14]$ for the Radon transform and its dual; and in [9] for the spherical mean operator and its dual on $\mathbb{R}$. Especially:

- We define and study the complex powers for the Dunkl Laplacian $\Delta_{\alpha}=\Lambda_{\alpha}^{2}$.

- We give inversion formulas for $S_{\alpha, \beta}$ and ${ }^{t} S_{\alpha, \beta}$ associated with integro-differential and integro-differential-difference operators when applied to some Lizorkin spaces of functions (see $[9,1,13])$.

- We establish a Plancherel formula for the operator ${ }^{t} S_{\alpha, \beta}$.

The content of this work is the following. In Section 2, we recall some results about the Dunkl operators. In particular, we give some properties of the operators $S_{\alpha, \beta}$ and ${ }^{t} S_{\alpha, \beta}$.

\footnotetext{
${ }^{\star}$ This paper is a contribution to the Special Issue on Dunkl Operators and Related Topics. The full collection is available at http://www.emis.de/journals/SIGMA/Dunkl_operators.html
} 
In Section 3, we consider the tempered distribution $|x|^{\lambda}$ for $\lambda \in \mathbb{C} \backslash\{-(\ell+1), \ell \in \mathbb{N}\}$ defined by

$$
\left\langle|x|^{\lambda}, \varphi\right\rangle:=\int_{\mathbb{R}}|x|^{\lambda} \varphi(x) d x
$$

Also we study the complex powers of the Dunkl Laplacian $\left(-\Delta_{\alpha}\right)^{\lambda}$, for some complex number $\lambda$. In the classical case when $\alpha=-1 / 2$, the complex powers of the usual Laplacian are given in [16].

In Section 4, we give the following inversion formulas:

$$
g=S_{\alpha, \beta} K_{1}\left({ }^{t} S_{\alpha, \beta}\right)(g), \quad f=\left({ }^{t} S_{\alpha, \beta}\right) K_{2} S_{\alpha, \beta}(f),
$$

where

$$
K_{1}(f)=\frac{c_{\beta}}{c_{\alpha}}\left(-\Delta_{\alpha}\right)^{\beta-\alpha} f, \quad K_{2}(f)=\frac{c_{\beta}}{c_{\alpha}}\left(-\Delta_{\beta}\right)^{\beta-\alpha} f \quad \text { and } \quad c_{\alpha}=\frac{1}{\left[2^{\alpha+1} \Gamma(\alpha+1)\right]^{2}} .
$$

Next, we give the following Plancherel formula for the operator ${ }^{t} S_{\alpha, \beta}$ :

$$
\int_{\mathbb{R}}|f(x)|^{2}|x|^{2 \beta+1} d x=\int_{\mathbb{R}}\left|K_{3}\left({ }^{t} S_{\alpha, \beta}(f)\right)(y)\right|^{2}|x|^{2 \alpha+1} d y,
$$

where

$$
K_{3}(f)=\sqrt{\frac{c_{\beta}}{c_{\alpha}}}\left(-\Delta_{\alpha}\right)^{(\beta-\alpha) / 2} f .
$$

\section{The Dunkl intertwining operator and its dual}

We consider the Dunkl operator $\Lambda_{\alpha}, \alpha \geq-1 / 2$, associated with the reflection group $\mathbb{Z}_{2}$ on $\mathbb{R}$ :

$$
\Lambda_{\alpha} f(x):=\frac{d}{d x} f(x)+\frac{2 \alpha+1}{x}\left[\frac{f(x)-f(-x)}{2}\right] .
$$

For $\alpha \geq-1 / 2$ and $\lambda \in \mathbb{C}$, the initial problem:

$$
\Lambda_{\alpha} f(x)=\lambda f(x), \quad f(0)=1,
$$

has a unique analytic solution $E_{\alpha}(\lambda x)$ called Dunkl kernel $[3,5]$ given by

$$
E_{\alpha}(\lambda x)=\Im_{\alpha}(\lambda x)+\frac{\lambda x}{2(\alpha+1)} \Im_{\alpha+1}(\lambda x),
$$

where

$$
\Im_{\alpha}(\lambda x):=\Gamma(\alpha+1) \sum_{n=0}^{\infty} \frac{(\lambda x / 2)^{2 n}}{n ! \Gamma(n+\alpha+1)},
$$

is the modified spherical Bessel function of order $\alpha$.

Notice that in the case $\alpha=-1 / 2$, we have

$$
\Lambda_{-1 / 2}=d / d x \quad \text { and } \quad E_{-1 / 2}(\lambda x)=e^{\lambda x} .
$$

For $\lambda \in \mathbb{C}$ and $x \in \mathbb{R}$, the Dunkl kernel $E_{\alpha}$ has the following Bochner-type representation $($ see $[3,11])$ :

$$
E_{\alpha}(\lambda x)=a_{\alpha} \int_{-1}^{1} e^{\lambda x t}\left(1-t^{2}\right)^{\alpha-1 / 2}(1+t) d t,
$$


where

$$
a_{\alpha}=\frac{\Gamma(\alpha+1)}{\sqrt{\pi} \Gamma(\alpha+1 / 2)},
$$

which can be written as:

$$
\begin{aligned}
& E_{\alpha}(\lambda x)=a_{\alpha} \operatorname{sgn}(x)|x|^{-(2 \alpha+1)} \int_{-|x|}^{|x|} e^{\lambda y}\left(x^{2}-y^{2}\right)^{\alpha-1 / 2}(x+y) d y, \quad x \neq 0, \\
& E_{\alpha}(0)=1 .
\end{aligned}
$$

We notice that, the Dunkl kernel $E_{\alpha}(\lambda x)$ can be also expanded in a power series [10] in the form:

$$
E_{\alpha}(\lambda x)=\sum_{n=0}^{\infty} \frac{(\lambda x)^{n}}{b_{n}(\alpha)}
$$

where

$$
b_{2 n}(\alpha)=\frac{2^{2 n} n !}{\Gamma(\alpha+1)} \Gamma(n+\alpha+1), \quad b_{2 n+1}(\alpha)=2(\alpha+1) b_{2 n}(\alpha+1) .
$$

Let $\alpha>-1 / 2$ and we define the Dunkl intertwining operator $V_{\alpha}$ on $\mathcal{E}(\mathbb{R})$ (the space of $C^{\infty}$-functions on $\left.\mathbb{R}\right)$, by

$$
V_{\alpha}(f)(x):=a_{\alpha} \int_{-1}^{1} f(x t)\left(1-t^{2}\right)^{\alpha-1 / 2}(1+t) d t,
$$

which can be written as:

$$
\begin{aligned}
& V_{\alpha}(f)(x)=a_{\alpha} \operatorname{sgn}(x)|x|^{-(2 \alpha+1)} \int_{-|x|}^{|x|} f(y)\left(x^{2}-y^{2}\right)^{\alpha-1 / 2}(x+y) d y, \quad x \neq 0, \\
& V_{\alpha}(f)(0)=f(0) .
\end{aligned}
$$

Remark 1. For $\alpha>-1 / 2$, we have

$$
E_{\alpha}(\lambda .)=V_{\alpha}\left(e^{\lambda \cdot}\right), \quad \lambda \in \mathbb{C} .
$$

Proposition 1 (see [18], Theorem 6.3). The operator $V_{\alpha}$ is a topological automorphism of $\mathcal{E}(\mathbb{R})$, and satisfies the transmutation relation:

$$
\Lambda_{\alpha}\left(V_{\alpha}(f)\right)=V_{\alpha}\left(\frac{d}{d x} f\right), \quad f \in \mathcal{E}(\mathbb{R}) .
$$

Let $\alpha>-1 / 2$ and we define the dual Dunkl intertwining operator ${ }^{t} V_{\alpha}$ on $\mathcal{S}(\mathbb{R})$ (the Schwartz space on $\mathbb{R})$, by

$$
{ }^{t} V_{\alpha}(f)(x):=a_{\alpha} \int_{|y| \geq|x|} \operatorname{sgn}(y)\left(y^{2}-x^{2}\right)^{\alpha-1 / 2}(x+y) f(y) d y,
$$

which can be written as:

$$
{ }^{t} V_{\alpha}(f)(x)=a_{\alpha} \operatorname{sgn}(x)|x|^{2 \alpha+1} \int_{|t| \geq 1} \operatorname{sgn}(t)\left(t^{2}-1\right)^{\alpha-1 / 2}(1+t) f(x t) d t .
$$


Proposition 2 (see [19], Theorems 3.2, 3.3).

(i) The operator ${ }^{t} V_{\alpha}$ is a topological automorphism of $\mathcal{S}(\mathbb{R})$, and satisfies the transmutation relation:

$$
{ }^{t} V_{\alpha}\left(\Lambda_{\alpha} f\right)=\frac{d}{d x}\left({ }^{t} V_{\alpha}(f)\right), \quad f \in \mathcal{S}(\mathbb{R}) .
$$

(ii) For all $f \in \mathcal{E}(\mathbb{R})$ and $g \in \mathcal{S}(\mathbb{R})$, we have

$$
\int_{\mathbb{R}} V_{\alpha}(f)(x) g(x)|x|^{2 \alpha+1} d x=\int_{\mathbb{R}} f(x)^{t} V_{\alpha}(g)(x) d x .
$$

Remark 2 (see [15]).

(i) For $\alpha>-1 / 2$ and $f \in \mathcal{E}(\mathbb{R})$, we can write

$$
V_{\alpha}(f)(x)=\Re_{\alpha}\left(f_{e}\right)(|x|)+\frac{1}{x} \Re_{\alpha}\left(M f_{o}\right)(|x|),
$$

where

$$
f_{e}(x)=\frac{1}{2}(f(x)+f(-x)), \quad f_{o}(x)=\frac{1}{2}(f(x)-f(-x)), \quad M f_{o}(x)=x f_{o}(x),
$$

and $\Re_{\alpha}$ is the Riemann-Liouville transform (see [17], page 75) given by

$$
\Re_{\alpha}\left(f_{e}\right)(x):=2 a_{\alpha} \int_{0}^{1} f_{e}(x t)\left(1-t^{2}\right)^{\alpha-1 / 2} d t, \quad x \geq 0 .
$$

Thus, we obtain

$$
V_{\alpha}^{-1}(f)(x)=\Re_{\alpha}^{-1}\left(f_{e}\right)(|x|)+\frac{1}{x} \Re_{\alpha}^{-1}\left(M f_{o}\right)(|x|) .
$$

Therefore (see also [20], Proposition 2.2), we get

$$
\begin{aligned}
& V_{\alpha}^{-1}\left(f_{e}\right)(x)=d_{\alpha} \frac{d}{d x}\left(\frac{d}{x d x}\right)^{r}\left\{x^{2 r+1} \int_{0}^{1} f_{e}(x t)\left(1-t^{2}\right)^{r-\alpha-1 / 2} t^{2 \alpha+1} d t\right\}, \\
& V_{\alpha}^{-1}\left(f_{o}\right)(x)=d_{\alpha}\left(\frac{d}{x d x}\right)^{r+1}\left\{x^{2 r+2} \int_{0}^{1} f_{o}(x t)\left(1-t^{2}\right)^{r-\alpha-1 / 2} t^{2 \alpha+2} d t\right\}
\end{aligned}
$$

where $r=[\alpha+1 / 2]$ denote the integer part of $\alpha+1 / 2$, and $d_{\alpha}=\frac{2^{-r} \pi}{\Gamma(\alpha+1) \Gamma(r-\alpha+1 / 2)}$.

(ii) For $\alpha>-1 / 2$ and $f \in \mathcal{S}(\mathbb{R})$, we can write

$$
{ }^{t} V_{\alpha}(f)(x)=W_{\alpha}\left(f_{e}\right)(|x|)+x W_{\alpha}\left(M^{-1} f_{o}\right)(|x|),
$$

where

$$
M^{-1} f_{o}(x)=\frac{1}{2 x}(f(x)-f(-x)),
$$

and $W_{\alpha}$ is the Weyl integral transform (see [17, page 85]) given by

$$
W_{\alpha}\left(f_{e}\right)(x):=2 a_{\alpha} x^{2 \alpha+1} \int_{1}^{\infty} f_{e}(x t)\left(t^{2}-1\right)^{\alpha-1 / 2} t d t, \quad x \geq 0 .
$$

Thus, we obtain

$$
\left({ }^{t} V_{\alpha}\right)^{-1} f(x)=W_{\alpha}^{-1}\left(f_{e}\right)(|x|)+x W_{\alpha}^{-1}\left(M^{-1} f_{o}\right)(|x|) .
$$


The Dunkl kernel gives rise to an integral transform, called Dunkl transform on $\mathbb{R}$, which was introduced by Dunkl in [4], where already many basic properties were established. Dunkl's results were completed and extended later on by de Jeu in [5].

The Dunkl transform of a function $f \in \mathcal{S}(\mathbb{R})$, is given by

$$
\mathcal{F}_{\alpha}(f)(\lambda):=\int_{\mathbb{R}} E_{\alpha}(-i \lambda x) f(x)|x|^{2 \alpha+1} d x, \quad \lambda \in \mathbb{R} .
$$

We notice that $\mathcal{F}_{-1 / 2}$ agrees with the Fourier transform $\mathcal{F}$ that is given by:

$$
\mathcal{F}(f)(\lambda):=\int_{\mathbb{R}} e^{-i \lambda x} f(x) d x, \quad \lambda \in \mathbb{R} .
$$

Proposition 3 (see [5]).

(i) For all $f \in \mathcal{S}(\mathbb{R})$, we have

$$
\mathcal{F}_{\alpha}\left(\Lambda_{\alpha} f\right)(\lambda)=i \lambda \mathcal{F}_{\alpha}(f)(\lambda), \quad \lambda \in \mathbb{R},
$$

where $\Lambda_{\alpha}$ is the Dunkl operator given by (1).

(ii) $\mathcal{F}_{\alpha}$ possesses on $\mathcal{S}(\mathbb{R})$ the following decomposition:

$$
\mathcal{F}_{\alpha}(f)=\mathcal{F} \circ{ }^{t} V_{\alpha}(f), \quad f \in \mathcal{S}(\mathbb{R}) .
$$

(iii) $\mathcal{F}_{\alpha}$ is a topological automorphism of $\mathcal{S}(\mathbb{R})$, and for $f \in \mathcal{S}(\mathbb{R})$ we have

$$
f(x)=c_{\alpha} \int_{\mathbb{R}} E_{\alpha}(i \lambda x) \mathcal{F}_{\alpha}(f)(\lambda)|\lambda|^{2 \alpha+1} d \lambda,
$$

where

$$
c_{\alpha}=\frac{1}{\left[2^{\alpha+1} \Gamma(\alpha+1)\right]^{2}} .
$$

(iv) The normalized Dunkl transform $\sqrt{c_{\alpha}} \mathcal{F}_{\alpha}$ extends uniquely to an isometric isomorphism of $L^{2}\left(\mathbb{R},|x|^{2 \alpha+1} d x\right)$ onto itself. In particular,

$$
\int_{\mathbb{R}}|f(x)|^{2}|x|^{2 \alpha+1} d x=c_{\alpha} \int_{\mathbb{R}}\left|\mathcal{F}_{\alpha}(f)(\lambda)\right|^{2}|\lambda|^{2 \alpha+1} d \lambda .
$$

For $T \in \mathcal{S}^{\prime}(\mathbb{R})$, we define the Dunkl transform $\mathcal{F}_{\alpha}(T)$ of $T$, by

$$
\left\langle\mathcal{F}_{\alpha}(T), \varphi\right\rangle:=\left\langle T, \mathcal{F}_{\alpha}(\varphi)\right\rangle, \quad \varphi \in \mathcal{S}(\mathbb{R}) .
$$

Thus the transform $\mathcal{F}_{\alpha}$ extends to a topological automorphism on $\mathcal{S}^{\prime}(\mathbb{R})$.

In [19], the author defines:

- The Dunkl translation operators $\tau_{x}, x \in \mathbb{R}$, on $\mathcal{E}(\mathbb{R})$, by

$$
\tau_{x} f(y):=\left(V_{\alpha}\right)_{x} \otimes\left(V_{\alpha}\right)_{y}\left[\left(V_{\alpha}\right)^{-1}(f)(x+y)\right], \quad y \in \mathbb{R} .
$$

These operators satisfy for $x, y \in \mathbb{R}$ and $\lambda \in \mathbb{C}$ the following properties:

$$
\begin{aligned}
& E_{\alpha}(\lambda x) E_{\alpha}(\lambda y)=\tau_{x}\left(E_{\alpha}(\lambda .)\right)(y), \quad \text { and } \\
& \mathcal{F}_{\alpha}\left(\tau_{x} f\right)(\lambda)=E_{k}(i \lambda x) \mathcal{F}_{\alpha}(f)(\lambda), \quad f \in \mathcal{S}(\mathbb{R}) .
\end{aligned}
$$


Proposition 4 (see [11]). If $f \in \mathcal{C}(\mathbb{R})$ (the space of continuous functions on $\mathbb{R}$ ) and $x, y \in \mathbb{R}$ such that $(x, y) \neq(0,0)$, then

$$
\begin{aligned}
& \tau_{x} f(y)=a_{\alpha} \int_{0}^{\pi}\left[f_{e}\left((x, y)_{\theta}\right)+f_{o}\left((x, y)_{\theta}\right) \frac{x+y}{(x, y)_{\theta}}\right][1-\operatorname{sgn}(x y) \cos \theta] \sin ^{2 \alpha} \theta d \theta, \\
& f_{e}(z)=\frac{1}{2}(f(z)+f(-z)), \quad f_{o}(z)=\frac{1}{2}(f(z)-f(-z)) \\
& (x, y)_{\theta}=\sqrt{x^{2}+y^{2}-2|x y| \cos \theta} .
\end{aligned}
$$

- The Dunkl convolution product $*_{\alpha}$ of two functions $f$ and $g$ in $\mathcal{S}(\mathbb{R})$, by

$$
f *_{\alpha} g(x):=\int_{\mathbb{R}} \tau_{x} f(-y) g(y)|y|^{2 \alpha+1} d y, \quad x \in \mathbb{R} .
$$

This convolution is associative, commutative in $\mathcal{S}(\mathbb{R})$ and satisfies (see [19, Theorem 7.2]):

$$
\mathcal{F}_{\alpha}\left(f *_{\alpha} g\right)=\mathcal{F}_{\alpha}(f) \mathcal{F}_{\alpha}(g) .
$$

For $T \in \mathcal{S}^{\prime}(\mathbb{R})$ and $f \in \mathcal{S}(\mathbb{R})$, we define the Dunkl convolution product $T *_{\alpha} f$, by

$$
T *_{\alpha} f(x):=\left\langle T(y), \tau_{x} f(-y)\right\rangle, \quad x \in \mathbb{R} .
$$

Note that $*_{-1 / 2}$ agrees with the standard convolution $*$ :

$$
T * f(x):=\langle T(y), f(x-y)\rangle .
$$

\section{The Dunkl Sonine transform}

In this section we study the Dunkl Sonine transform, which also studied by Y. Xu on polynomials in [20]. For thus we consider the following identity, which is a consequence of Xu's result when we extend the result of Lemma 2.1 on $\mathcal{E}(\mathbb{R})$.

Proposition 5. Let $\alpha, \beta \in]-1 / 2, \infty[$, such that $\beta>\alpha$. Then

$$
E_{\beta}(\lambda x)=a_{\alpha, \beta} \int_{-1}^{1} E_{\alpha}(\lambda x t)\left(1-t^{2}\right)^{\beta-\alpha-1}(1+t)|t|^{2 \alpha+1} d t,
$$

where

$$
a_{\alpha, \beta}=\frac{\Gamma(\beta+1)}{\Gamma(\beta-\alpha) \Gamma(\alpha+1)} .
$$

Proof. From (2), we have

$$
\int_{-1}^{1} E_{\alpha}(\lambda x t)\left(1-t^{2}\right)^{\beta-\alpha-1}(1+t)|t|^{2 \alpha+1} d t=\sum_{n=0}^{\infty} \frac{(\lambda x)^{n}}{b_{n}(\alpha)} I_{n}(\alpha, \beta),
$$

where

$$
I_{n}(\alpha, \beta)=\int_{-1}^{1} t^{n}\left(1-t^{2}\right)^{\beta-\alpha-1}(1+t)|t|^{2 \alpha+1} d t,
$$

or

$$
I_{2 n}(\alpha, \beta)=2 \int_{0}^{1}\left(1-t^{2}\right)^{\beta-\alpha-1} t^{2 n+2 \alpha+1} d t=\int_{0}^{1}(1-y)^{\beta-\alpha-1} y^{n+\alpha} d y
$$




$$
=\frac{\Gamma(\beta-\alpha) \Gamma(n+\alpha+1)}{\Gamma(n+\beta+1)},
$$

and

$$
I_{2 n+1}(\alpha, \beta)=2 \int_{0}^{1}\left(1-t^{2}\right)^{\beta-\alpha-1} t^{2 n+2 \alpha+3} d t=I_{2 n}(\alpha+1, \beta+1) .
$$

Thus

$$
\int_{-1}^{1} E_{\alpha}(\lambda x t)\left(1-t^{2}\right)^{\beta-\alpha-1}(1+t)|t|^{2 \alpha+1} d t=\frac{\Gamma(\beta-\alpha) \Gamma(\alpha+1)}{\Gamma(\beta+1)} E_{\beta}(\lambda x),
$$

which gives the desired result.

Remark 3. We can write the formula (5) by the following

$$
E_{\beta}(\lambda x)=a_{\alpha, \beta} \operatorname{sgn}(x)|x|^{-(2 \beta+1)} \int_{-|x|}^{|x|} E_{\alpha}(\lambda y)\left(x^{2}-y^{2}\right)^{\beta-\alpha-1}(x+y)|y|^{2 \alpha+1} d y, \quad x \neq 0 .
$$

Definition 1. Let $\alpha, \beta \in]-1 / 2, \infty[$, such that $\beta>\alpha$. We define the Dunkl Sonine transform $S_{\alpha, \beta}$ on $\mathcal{E}(\mathbb{R})$, by

$$
S_{\alpha, \beta}(f)(x):=a_{\alpha, \beta} \int_{-1}^{1} f(x t)\left(1-t^{2}\right)^{\beta-\alpha-1}(1+t)|t|^{2 \alpha+1} d t,
$$

which can be written as:

$$
\begin{aligned}
& S_{\alpha, \beta}(f)(x)=a_{\alpha, \beta} \operatorname{sgn}(x)|x|^{-(2 \beta+1)} \int_{-|x|}^{|x|} f(y)\left(x^{2}-y^{2}\right)^{\beta-\alpha-1}(x+y)|y|^{2 \alpha+1} d y, \quad x \neq 0, \\
& S_{\alpha, \beta}(f)(0)=f(0) .
\end{aligned}
$$

Remark 4. For $\alpha, \beta \in]-1 / 2, \infty[$, such that $\beta>\alpha$, we have

$$
E_{\beta}(\lambda .)=S_{\alpha, \beta}\left(E_{\alpha}(\lambda .)\right), \quad \lambda \in \mathbb{C} .
$$

Definition 2. Let $\alpha, \beta \in]-1 / 2, \infty[$, such that $\beta>\alpha$. We define the dual Dunkl Sonine transform ${ }^{t} S_{\alpha, \beta}$ on $\mathcal{S}(\mathbb{R})$, by

$$
{ }^{t} S_{\alpha, \beta}(f)(x):=a_{\alpha, \beta} \int_{|y| \geq|x|} \operatorname{sgn}(y)\left(y^{2}-x^{2}\right)^{\beta-\alpha-1}(x+y) f(y) d y,
$$

which can be written as:

$$
{ }^{t} S_{\alpha, \beta}(f)(x)=a_{\alpha, \beta} \operatorname{sgn}(x)|x|^{2(\beta-\alpha)} \int_{|t| \geq 1} \operatorname{sgn}(t)\left(t^{2}-1\right)^{\beta-\alpha-1}(t+1) f(x t) d t .
$$

\section{Proposition 6.}

(i) For all $f \in \mathcal{E}(\mathbb{R})$ and $g \in \mathcal{S}(\mathbb{R})$, we have

$$
\int_{\mathbb{R}} S_{\alpha, \beta}(f)(x) g(x)|x|^{2 \beta+1} d x=\int_{\mathbb{R}} f(x)^{t} S_{\alpha, \beta}(g)(x)|x|^{2 \alpha+1} d x .
$$

(ii) $\mathcal{F}_{\beta}$ possesses on $\mathcal{S}(\mathbb{R})$ the following decomposition:

$$
\mathcal{F}_{\beta}(f)=\mathcal{F}_{\alpha} \circ{ }^{t} S_{\alpha, \beta}(f), \quad f \in \mathcal{S}(\mathbb{R}) .
$$


Proof. Part $(i)$ follows from Definition 1 by Fubini's theorem. Then part $(i i)$ follows from $(i)$ and (6) by taking $f=E_{\alpha}(-i \lambda$.).

In [20, Lemma 2.1] Y. Xu proves the identity $S_{\alpha, \beta}=V_{\beta} \circ V_{\alpha}^{-1}$ on polynomials. As the intertwiner is a homeomorphism on $\mathcal{E}(\mathbb{R})$ and polynomials are dense in $\mathcal{E}(\mathbb{R})$, this gives the identity also on $\mathcal{E}(\mathbb{R})$. In the following we give a second method to prove this identity.

\section{Theorem 1.}

(i) The operator ${ }^{t} S_{\alpha, \beta}$ is a topological automorphism of $\mathcal{S}(\mathbb{R})$, and satisfies the following relations:

$$
\begin{aligned}
& { }^{t} S_{\alpha, \beta}(f)=\left({ }^{t} V_{\alpha}\right)-1 \circ{ }^{t} V_{\beta}(f), \quad f \in \mathcal{S}(\mathbb{R}), \\
& { }^{t} S_{\alpha, \beta}\left(\Lambda_{\beta} f\right)=\Lambda_{\alpha}\left({ }^{t} S_{\alpha, \beta}(f)\right), \quad f \in \mathcal{S}(\mathbb{R}) .
\end{aligned}
$$

(ii) The operator $S_{\alpha, \beta}$ is a topological automorphism of $\mathcal{E}(\mathbb{R})$, and satisfies the following relations:

$$
\begin{array}{ll}
S_{\alpha, \beta}(f)=V_{\beta} \circ V_{\alpha}^{-1}(f), & f \in \mathcal{E}(\mathbb{R}), \\
\Lambda_{\beta}\left(S_{\alpha, \beta}(f)\right)=S_{\alpha, \beta}\left(\Lambda_{\alpha} f\right), & f \in \mathcal{E}(\mathbb{R}) .
\end{array}
$$

Proof. (i) From Proposition 6 (ii), we have

$$
{ }^{t} S_{\alpha, \beta}(f)=\left(\mathcal{F}_{\alpha}\right)^{-1} \circ \mathcal{F}_{\beta}(f) .
$$

Using Proposition $3(i i)$, we obtain

$$
{ }^{t} S_{\alpha, \beta}(f)=\left({ }^{t} V_{\alpha}\right){ }^{-1} \circ{ }^{t} V_{\beta}(f), \quad f \in \mathcal{S}(\mathbb{R}) .
$$

Thus from Proposition $2(i)$,

$$
{ }^{t} S_{\alpha, \beta}\left(\Lambda_{\beta} f\right)=\left({ }^{t} V_{\alpha}\right)^{-1} \circ{ }^{t} V_{\beta}\left(\Lambda_{\beta} f\right)=\left({ }^{t} V_{\alpha}\right)^{-1}\left(\frac{d}{d x}{ }^{t} V_{\beta}(f)\right) .
$$

Using the fact that

$$
{ }^{t} V_{\alpha}\left(\Lambda_{\alpha} f\right)=\frac{d}{d x}\left({ }^{t} V_{\alpha}(f)\right) \Longleftrightarrow \Lambda_{\alpha}\left({ }^{t} V_{\alpha}\right)^{-1}(f)=\left({ }^{t} V_{\alpha}\right)^{-1}\left(\frac{d}{d x} f\right),
$$

we obtain

$$
{ }^{t} S_{\alpha, \beta}\left(\Lambda_{\beta} f\right)=\Lambda_{\alpha}\left({ }^{t} V_{\alpha}\right){ }^{-1}\left({ }^{t} V_{\beta}(f)\right)=\Lambda_{\alpha}\left({ }^{t} S_{\alpha, \beta}(f)\right) .
$$

(ii) From Proposition 2 (ii), we have

$$
\int_{\mathbb{R}} f(x)^{t} V_{\beta}(g)(x) d x=\int_{\mathbb{R}} V_{\beta}(f)(x) g(x)|x|^{2 \beta+1} d x .
$$

On other hand, from (8), Proposition 2 (ii) and Proposition $6(i)$ we have

$$
\begin{aligned}
\int_{\mathbb{R}} f(x){ }^{t} V_{\beta}(g)(x) d x & =\int_{\mathbb{R}} f(x){ }^{t} V_{\alpha} \circ{ }^{t} S_{\alpha, \beta}(g)(x) d x=\int_{\mathbb{R}} V_{\alpha}(f)(x){ }^{t} S_{\alpha, \beta}(g)(x)|x|^{2 \alpha+1} d x \\
& =\int_{\mathbb{R}} S_{\alpha, \beta} \circ V_{\alpha}(f)(x) g(x)|x|^{2 \beta+1} d x .
\end{aligned}
$$

Then

$$
S_{\alpha, \beta} \circ V_{\alpha}(f)=V_{\beta}(f) .
$$


Hence from Proposition 1,

$$
\Lambda_{\beta}\left(S_{\alpha, \beta}(f)\right)=\Lambda_{\beta} V_{\beta}\left(V_{\alpha}^{-1}(f)\right)=V_{\beta}\left(\frac{d}{d x} V_{\alpha}^{-1}(f)\right) .
$$

Using the fact that

$$
\Lambda_{\alpha}\left(V_{\alpha}(f)\right)=V_{\alpha}\left(\frac{d}{d x} f\right) \Longleftrightarrow V_{\alpha}^{-1}\left(\Lambda_{\alpha} f\right)=\frac{d}{d x} V_{\alpha}^{-1}(f)
$$

we obtain

$$
\Lambda_{\beta}\left(S_{\alpha, \beta}(f)\right)=V_{\beta} \circ V_{\alpha}^{-1}\left(\Lambda_{\alpha} f\right)=S_{\alpha, \beta}\left(\Lambda_{\alpha} f\right),
$$

which completes the proof of the theorem.

\section{Complex powers of $\Delta_{\alpha}$}

For $\lambda \in \mathbb{C}, \operatorname{Re}(\lambda)>-1$, we denote by $|x|^{\lambda}$ the tempered distribution defined by

$$
\left\langle|x|^{\lambda}, \varphi\right\rangle:=\int_{\mathbb{R}}|x|^{\lambda} \varphi(x) d x, \quad \varphi \in \mathcal{S}(\mathbb{R})
$$

We write

$$
\left\langle|x|^{\lambda}, \varphi\right\rangle=\int_{0}^{\infty} x^{\lambda}[\varphi(x)+\varphi(-x)] d x, \quad \varphi \in \mathcal{S}(\mathbb{R}),
$$

then from [1], we obtain the following result.

Lemma 1. Let $\varphi \in \mathcal{S}(\mathbb{R})$. The mapping $g: \lambda \rightarrow\left\langle|x|^{\lambda}, \varphi\right\rangle$ is complex-valued function and has an analytic extension to $\mathbb{C} \backslash\{-(1+2 \ell), \ell \in \mathbb{N}\}$, with simple poles $-(2 \ell+1), \ell \in \mathbb{N}$ and

$$
\operatorname{Res}(g,-1-2 \ell)=2 \frac{\varphi^{(2 \ell)}(0)}{(2 \ell) !}
$$

Proposition 7. Let $\varphi \in \mathcal{S}(\mathbb{R})$.

(i) The function $\lambda \rightarrow\left\langle|x|^{\lambda+2 \alpha+1}, \varphi\right\rangle$ is analytic on $\mathbb{C} \backslash\{-(2 \alpha+2 \ell+2), \ell \in \mathbb{N}\}$, with simple poles $-(2 \alpha+2 \ell+2), \ell \in \mathbb{N}$.

(ii) The function $\lambda \rightarrow \frac{2^{2 \alpha+\lambda+2} \Gamma(\alpha+1) \Gamma\left(\frac{2 \alpha+\lambda+2}{2}\right)}{\Gamma(-\lambda / 2)}\left\langle|x|^{-(\lambda+1)}, \varphi\right\rangle$ is analytic on $\mathbb{C} \backslash\{-(2 \alpha+2 \ell+2)$, $\ell \in \mathbb{N}\}$, with simple poles $-(2 \alpha+2 \ell+2), \ell \in \mathbb{N}$.

(iii) For $\lambda \in \mathbb{C} \backslash\{-(2 \alpha+2 \ell+2), \ell \in \mathbb{N}\}$ we have

$$
\mathcal{F}_{\alpha}\left(|x|^{\lambda+2 \alpha+1}\right)=\frac{2^{2 \alpha+\lambda+2} \Gamma(\alpha+1) \Gamma\left(\frac{2 \alpha+\lambda+2}{2}\right)}{\Gamma(-\lambda / 2)}|x|^{-(\lambda+1)}, \quad \text { in } \mathcal{S}^{\prime} \text {-sense. }
$$

(iv) For $\lambda \in \mathbb{C} \backslash\{-(2 \alpha+2 \ell+2), \ell \in \mathbb{N}\}$ we have

$$
|x|^{\lambda+2 \alpha+1}=\frac{2^{\lambda} \Gamma\left(\frac{2 \alpha+\lambda+2}{2}\right)}{\Gamma(\alpha+1) \Gamma(-\lambda / 2)} \mathcal{F}_{\alpha}\left(|x|^{-(\lambda+1)}\right), \quad \text { in } \mathcal{S}^{\prime} \text {-sense. }
$$


Proof. (i) Follows directly from Lemma 1.

(ii) From [7, pages 2 and 8] the function $\lambda \rightarrow \Gamma\left(\frac{2 \alpha+\lambda+2}{2}\right)$ has an analytic extension to $\mathbb{C} \backslash\{-(2 \alpha+2 \ell+2), \ell \in \mathbb{N}\}$, with simple poles $-(2 \alpha+2 \ell+2), \ell \in \mathbb{N}$, and the function $\lambda \rightarrow \frac{1}{\Gamma(-\lambda / 2)}$ has zeros $2 \ell, \ell \in \mathbb{N}$. Thus from Lemma 1 we see that

$$
\lambda \rightarrow \frac{2^{2 \alpha+\lambda+2} \Gamma(\alpha+1) \Gamma\left(\frac{2 \alpha+\lambda+2}{2}\right)}{\Gamma(-\lambda / 2)}\left\langle|x|^{-(\lambda+1)}, \varphi\right\rangle
$$

is analytic on $\mathbb{C} \backslash\{-(2 \alpha+2 \ell+2), \ell \in \mathbb{N}\}$, with simple poles $-(2 \alpha+2 \ell+2), \ell \in \mathbb{N}$.

(iii) Let determine the value of $\mathcal{F}_{\alpha}\left(|x|^{\lambda+2 \alpha+1}\right)$ in the $\mathcal{S}^{\prime}$-sense. We put $\psi_{t}(x):=e^{-t x^{2}}, t>0$. Then $\psi_{t} \in \mathcal{S}(\mathbb{R})$, and from [12]:

$$
\mathcal{F}_{\alpha}\left(\psi_{t}\right)(x)=\Gamma(\alpha+1) t^{-(\alpha+1)} e^{-x^{2} / 4 t}, \quad x \in \mathbb{R} .
$$

Furthermore, for $\varphi \in \mathcal{S}(\mathbb{R})$ we have

$$
\int_{\mathbb{R}} \mathcal{F}_{\alpha}(\varphi)(x) \psi_{t}(x)|x|^{2 \alpha+1} d x=\Gamma(\alpha+1) \int_{\mathbb{R}} \varphi(x) t^{-(\alpha+1)} e^{-x^{2} / 4 t}|x|^{2 \alpha+1} d x .
$$

Multiplying both sides by $t^{-\lambda / 2-1}$ and integrating over $(0, \infty)$, we obtain for $\left.\operatorname{Re}(\lambda) \in\right]-(2 \alpha+$ 2), $0[$ :

$$
\int_{\mathbb{R}} \mathcal{F}_{\alpha}(\varphi)(x)|x|^{\lambda+2 \alpha+1} d x=\frac{2^{2 \alpha+\lambda+2} \Gamma(\alpha+1) \Gamma\left(\frac{2 \alpha+\lambda+2}{2}\right)}{\Gamma(-\lambda / 2)} \int_{\mathbb{R}} \varphi(x)|x|^{-(\lambda+1)} d x .
$$

This and from (3) we get for $\operatorname{Re}(\lambda) \in]-(2 \alpha+2), 0[$ :

$$
\mathcal{F}_{\alpha}\left(|x|^{\lambda+2 \alpha+1}\right)=\frac{2^{2 \alpha+\lambda+2} \Gamma(\alpha+1) \Gamma\left(\frac{2 \alpha+\lambda+2}{2}\right)}{\Gamma(-\lambda / 2)}|x|^{-(\lambda+1)} .
$$

The result follows by analytic continuation.

(iv) From (iii) we have

$$
|x|^{\lambda+2 \alpha+1}=\frac{2^{2 \alpha+\lambda+2} \Gamma(\alpha+1) \Gamma\left(\frac{2 \alpha+\lambda+2}{2}\right)}{\Gamma(-\lambda / 2)} \mathcal{F}_{\alpha}^{-1}\left(|x|^{-(\lambda+1)}\right) .
$$

Using the fact that

$$
\left\langle\mathcal{F}_{\alpha}^{-1}\left(|x|^{-(\lambda+1)}\right), \varphi\right\rangle=\left\langle|x|^{-(\lambda+1)}, \mathcal{F}_{\alpha}^{-1}(\varphi)\right\rangle, \quad \varphi \in \mathcal{S}(\mathbb{R}) .
$$

By applying (9) and Proposition 3 (iii), we obtain

$$
\left\langle\mathcal{F}_{\alpha}^{-1}\left(|x|^{-(\lambda+1)}\right), \varphi\right\rangle=c_{\alpha} \int_{\mathbb{R}}|x|^{-(\lambda+1)} \mathcal{F}_{\alpha}(\varphi)(-x) d x, \quad \varphi \in \mathcal{S}(\mathbb{R}) .
$$

Then

$$
\mathcal{F}_{\alpha}^{-1}\left(|x|^{-(\lambda+1)}\right)=c_{\alpha} \mathcal{F}_{\alpha}\left(|x|^{-(\lambda+1)}\right),
$$

which gives the result.

Definition 3. For $\lambda \in \mathbb{C} \backslash\{-(\alpha+\ell+1), \ell \in \mathbb{N}\}$, the complex powers of the Dunkl Laplacian $\Delta_{\alpha}$ are defined for $f \in \mathcal{S}(\mathbb{R})$ by

$$
\left(-\Delta_{\alpha}\right)^{\lambda} f(x):=\frac{2^{2 \lambda} \Gamma(\alpha+\lambda+1)}{\Gamma(\alpha+1) \Gamma(-\lambda)}|x|^{-(2 \lambda+1)} *_{\alpha} f(x),
$$

where $*_{\alpha}$ is the Dunkl convolution product given by (4). 
In the next part of this section we use Definition 3 and Proposition 7 (iv) to establish the following result:

$$
\mathcal{F}_{\alpha}\left(\left(-\Delta_{\alpha}\right)^{\lambda} f\right)(x)=|x|^{2 \lambda} \mathcal{F}_{\alpha}(f)(x) .
$$

Proposition 8. For $\lambda \in \mathbb{C} \backslash\{-(\alpha+\ell+1), \ell \in \mathbb{N}\}$ and $f \in \mathcal{S}(\mathbb{R})$,

$$
\left(-\Delta_{\alpha}\right)^{\lambda} f(x)=b_{\alpha}(\lambda) \int_{\mathbb{R}}\left[\int_{0}^{\pi} \frac{(1+\operatorname{sgn}(x y) \cos \theta)}{(x, y)_{\theta}^{2(\lambda+\alpha+1)}} \sin ^{2 \alpha} \theta d \theta\right] f(y)|y|^{2 \alpha+1} d y,
$$

where

$$
b_{\alpha}(\lambda)=\frac{2^{2 \lambda} \Gamma(\alpha+\lambda+1)}{\sqrt{\pi} \Gamma(\alpha+1 / 2) \Gamma(-\lambda)}, \quad(x, y)_{\theta}=\sqrt{x^{2}+y^{2}-2|x y| \cos \theta} .
$$

Proof. From Definition 3, (4) and (9), we have

$$
\begin{aligned}
\left(-\Delta_{\alpha}\right)^{\lambda} f(x) & =\frac{2^{2 \lambda} \Gamma(\alpha+\lambda+1)}{\Gamma(\alpha+1) \Gamma(-\lambda)}\left\langle|y|^{-(2 \lambda+1)}, \tau_{x} f(-y)\right\rangle \\
& =\frac{2^{2 \lambda} \Gamma(\alpha+\lambda+1)}{\Gamma(\alpha+1) \Gamma(-\lambda)} \int_{\mathbb{R}}|y|^{-2(\lambda+\alpha+1)} \tau_{x} f(-y)|y|^{2 \alpha+1} d y .
\end{aligned}
$$

So

$$
\left(-\Delta_{\alpha}\right)^{\lambda} f(x)=\int_{\mathbb{R}} \tau_{x}\left(|y|^{-2(\lambda+\alpha+1)}\right)(-y) f(y)|y|^{2 \alpha+1} d y
$$

Then the result follows from Proposition 4.

Note 1. We denote by

- $\Psi$ the subspace of $\mathcal{S}(\mathbb{R})$ consisting of functions $f$, such that

$$
f^{(k)}(0)=0, \quad \forall k \in \mathbb{N} .
$$

- $\Phi_{\alpha}$ the subspace of $\mathcal{S}(\mathbb{R})$ consisting of functions $f$, such that

$$
\int_{\mathbb{R}} f(y) y^{k}|y|^{2 \alpha+1} d y=0, \quad \forall k \in \mathbb{N} .
$$

The spaces $\Psi$ and $\Phi_{-1 / 2}$ are well-known in the literature as Lizorkin spaces (see $\left.[1,9,13]\right)$.

Lemma 2 (see [1]). The multiplication operator $M_{\lambda}: f \rightarrow|x|^{\lambda} f, \lambda \in \mathbb{C}$, is a topological automorphism of $\Psi$. Its inverse operator is $\left(M_{\lambda}\right)^{-1}=M_{-\lambda}$.

\section{Theorem 2.}

(i) The Dunkl transform $\mathcal{F}_{\alpha}$ is a topological isomorphism from $\Phi_{\alpha}$ onto $\Psi$.

(ii) The operator ${ }^{t} S_{\alpha, \beta}$ is a topological isomorphism from $\Phi_{\beta}$ onto $\Phi_{\alpha}$.

(iii) For $\lambda \in \mathbb{C} \backslash\{-(\alpha+\ell+1), \ell \in \mathbb{N}\}$ and $f \in \Phi_{\alpha}$, the function $\left(-\Delta_{\alpha}\right)^{\lambda} f$ belongs to $\in \Phi_{\alpha}$, and

$$
\mathcal{F}_{\alpha}\left(\left(-\Delta_{\alpha}\right)^{\lambda} f\right)(x)=|x|^{2 \lambda} \mathcal{F}_{\alpha}(f)(x)
$$


Proof. $(i)$ Let $f \in \Phi_{\alpha}$, then

$$
\left(\mathcal{F}_{\alpha}(f)\right)^{(k)}(0)=(-i)^{k} \frac{k !}{b_{k}(\alpha)} \int_{\mathbb{R}} f(x) x^{k}|x|^{2 \alpha+1} d y=0, \quad \forall k \in \mathbb{N} .
$$

Hence $\mathcal{F}_{\alpha}(f) \in \Psi$.

Conversely, let $g \in \Psi$. Since $\mathcal{F}_{\alpha}$ is a topological automorphism of $\mathcal{S}(\mathbb{R})$. There exists $f \in \mathcal{S}(\mathbb{R})$, such that $\mathcal{F}_{\alpha}(f)=g$. Thus

$$
g^{(k)}(0)=(-i)^{k} \frac{k !}{b_{k}(\alpha)} \int_{\mathbb{R}} f(x) x^{k}|x|^{2 \alpha+1} d y=0, \quad \forall k \in \mathbb{N} .
$$

So $f \in \Phi_{\alpha}$ and $\mathcal{F}_{\alpha}(f)=g$.

(ii) follows directly from $(i)$ and $(7)$.

(iii) Similarly to the standard convolution if $f \in \mathcal{S}(\mathbb{R})$ and $S \in \mathcal{S}^{\prime}(\mathbb{R})$, then $S *_{\alpha} f \in \mathcal{E}(\mathbb{R})$ and $T_{|x|^{2 \alpha+1} S *_{\alpha} f} \in \mathcal{S}^{\prime}(\mathbb{R})$. Moreover

$$
\mathcal{F}_{\alpha}\left(T_{|x|^{2 \alpha+1} S *_{\alpha} f}\right)=\mathcal{F}_{\alpha}(f) \mathcal{F}_{\alpha}(S) .
$$

Let $f \in \Phi_{\alpha}$ and $\lambda \in \mathbb{C} \backslash\{-(\alpha+\ell+1), \ell \in \mathbb{N}\}$. Consequently, from Definition 3, Proposition $7(i v)$ and (9) we have

$$
\mathcal{F}_{\alpha}\left(T_{|x|^{2 \alpha+1}\left(-\Delta_{\alpha}\right)^{\lambda} f}\right)=|x|^{2 \lambda+2 \alpha+1} \mathcal{F}_{\alpha}(f)=T_{|x|^{2 \lambda+2 \alpha+1} \mathcal{F}_{\alpha}(f)} .
$$

On the other hand from (3),

$$
\mathcal{F}_{\alpha}\left(T_{|x|^{2 \alpha+1}\left(-\Delta_{\alpha}\right)^{\lambda} f}\right)=T_{|x|^{2 \alpha+1} \mathcal{F}_{\alpha}\left(\left(-\Delta_{\alpha}\right)^{\lambda} f\right)} .
$$

From (11) and (12), we obtain

$$
\mathcal{F}_{\alpha}\left(\left(-\Delta_{\alpha}\right)^{\lambda} f\right)=|x|^{2 \lambda} \mathcal{F}_{\alpha}(f) .
$$

Then by Lemma 2 and $(i)$ we deduce that $\left(-\Delta_{\alpha}\right)^{\lambda} f \in \Phi_{\alpha}$.

\section{Inversion formulas for $S_{\alpha, \beta}$ and ${ }^{t} S_{\alpha, \beta}$}

In this section, we establish inversion formulas for the Dunkl Sonine transform and its dual.

Definition 4. We define the operators $K_{1}, K_{2}$ and $K_{3}$, by

$$
\begin{aligned}
K_{1}(f) & :=\frac{c_{\beta}}{c_{\alpha}} \mathcal{F}_{\alpha}^{-1}\left(|\lambda|^{2(\beta-\alpha)} \mathcal{F}_{\alpha}(f)\right)=\frac{c_{\beta}}{c_{\alpha}}\left(-\Delta_{\alpha}\right)^{\beta-\alpha} f, \quad f \in \Phi_{\alpha}, \\
K_{2}(f) & :=\frac{c_{\beta}}{c_{\alpha}} \mathcal{F}_{\beta}^{-1}\left(|\lambda|^{2(\beta-\alpha)} \mathcal{F}_{\beta}(f)\right)=\frac{c_{\beta}}{c_{\alpha}}\left(-\Delta_{\beta}\right)^{\beta-\alpha} f, \quad f \in \Phi_{\beta}, \\
K_{3}(f) & :=\sqrt{\frac{c_{\beta}}{c_{\alpha}}} \mathcal{F}_{\alpha}^{-1}\left(|\lambda|^{\beta-\alpha} \mathcal{F}_{\alpha}(f)\right)=\sqrt{\frac{c_{\beta}}{c_{\alpha}}}\left(-\Delta_{\alpha}\right)^{(\beta-\alpha) / 2} f, \quad f \in \Phi_{\alpha} .
\end{aligned}
$$

Lemma 3. For all $g \in \Phi_{\beta}$, we have

$$
K_{1}\left({ }^{t} S_{\alpha, \beta}\right)(g)=\left({ }^{t} S_{\alpha, \beta}\right) K_{2}(g) .
$$

Proof. Let $g \in \Phi_{\beta}$. Using Proposition 6 (ii),

$$
K_{1}\left({ }^{t} S_{\alpha, \beta}\right)(g)=\frac{c_{\beta}}{c_{\alpha}} \mathcal{F}_{\alpha}^{-1}\left(|\lambda|^{2(\beta-\alpha)} \mathcal{F}_{\beta}(g)\right)=\left({ }^{t} S_{\alpha, \beta}\right) K_{2}(g) .
$$




\section{Theorem 3.}

(i) Inversion formulas: For all $f \in \Phi_{\alpha}$ and $g \in \Phi_{\beta}$, we have the inversions formulas:
(a) $g=S_{\alpha, \beta} K_{1}\left({ }^{t} S_{\alpha, \beta}\right)(g)$,
(b) $f=\left({ }^{t} S_{\alpha, \beta}\right) K_{2} S_{\alpha, \beta}(f)$.

(ii) Plancherel formula: For all $f \in \Phi_{\beta}$ we have

$$
\int_{\mathbb{R}}|f(x)|^{2}|x|^{2 \beta+1} d x=\int_{\mathbb{R}}\left|K_{3}\left({ }^{t} S_{\alpha, \beta}(f)\right)(x)\right|^{2}|x|^{2 \alpha+1} d x .
$$

Proof. (i) Let $g \in \Phi_{\beta}$. From Proposition 3 (iii), (6) and Proposition 6 (ii), we obtain

$$
\begin{aligned}
g & =c_{\beta} \int_{\mathbb{R}} S_{\alpha, \beta}\left(E_{\alpha}(i \lambda .)\right) \mathcal{F}_{\beta}(g)(\lambda)|\lambda|^{2 \beta+1} d \lambda \\
& =c_{\beta} S_{\alpha, \beta}\left[\int_{\mathbb{R}} E_{\alpha}(i \lambda .) \mathcal{F}_{\alpha} \circ{ }^{t} S_{\alpha, \beta}(g)(\lambda)|\lambda|^{2 \beta+1} d \lambda\right] \\
& =\frac{c_{\beta}}{c_{\alpha}} S_{\alpha, \beta}\left[\mathcal{F}_{\alpha}^{-1}\left(|\lambda|^{2(\beta-\alpha)} \mathcal{F}_{\alpha} \circ{ }^{t} S_{\alpha, \beta}(g)\right)\right] .
\end{aligned}
$$

Thus

$$
g=S_{\alpha, \beta} K_{1}\left({ }^{t} S_{\alpha, \beta}\right)(g), \quad g \in \Phi_{\beta} .
$$

From the previous relation and (13), we deduce the relation:

$$
f=\left({ }^{t} S_{\alpha, \beta}\right) K_{2} S_{\alpha, \beta}(f), \quad f \in \Phi_{\alpha} .
$$

(ii) Let $f \in \Phi_{\beta}$. From Proposition $3(i v)$ and Proposition 6 (ii), we deduce that

$$
\int_{\mathbb{R}}|f(x)|^{2}|x|^{2 \beta+1} d x=\left.\left.c_{\beta} \int_{\mathbb{R}}|| \lambda\right|^{\beta-\alpha} \mathcal{F}_{\alpha}\left({ }^{t} S_{\alpha, \beta}(f)\right)(\lambda)\right|^{2}|\lambda|^{2 \alpha+1} d \lambda .
$$

Thus we obtain

$$
\int_{\mathbb{R}}|f(x)|^{2}|x|^{2 \beta+1} d x=c_{\alpha} \int_{\mathbb{R}}\left|\mathcal{F}_{\alpha}\left(K_{3}\left({ }^{t} S_{\alpha, \beta}(f)\right)\right)(\lambda)\right|^{2}|\lambda|^{2 \alpha+1} d \lambda .
$$

Then the result follows from this identity by applying Proposition 3 (iv).

Remark 5. Let $f \in \Phi_{\alpha}$ and $g \in \Phi_{\beta}$. By writing $(a)$ and (b) respectively for the functions $S_{\alpha, \beta}(f)$ and ${ }^{t} S_{\alpha, \beta}(g)$, we obtain
(c) $f=K_{1}\left({ }^{t} S_{\alpha, \beta}\right) S_{\alpha, \beta}(f)$,
(d) $g=K_{2} S_{\alpha, \beta}\left({ }^{t} S_{\alpha, \beta}\right)(g)$.

\section{Acknowledgements}

The author is very grateful to the referees and editors for many critical comments on this paper.

\section{References}

[1] Baccar C., Hamadi N.B., Rachdi L.T., Inversion formulas for Riemann-Liouville transform and its dual associated with singular partial differential operators, Int. J. Math. Math. Sci. 2006 (2006), Art. ID 86238, 26 pages.

[2] Dunkl C.F., Differential-difference operators associated with reflections groups, Trans. Amer. Math. Soc. 311 (1989), 167-183.

[3] Dunkl C.F., Integral kernels with reflection group invariance, Canad. J. Math. 43 (1991), 1213-1227. 
[4] Dunkl C.F., Hankel transforms associated to finite reflection groups, Contemp. Math. 138 (1992), $123-138$.

[5] de Jeu M.F.E., The Dunkl transform, Invent. Math. 113 (1993), 147-162.

[6] Lapointe L., Vinet L., Exact operator solution of the Calogero-Sutherland model, Comm. Math. Phys. 178 (1996), 425-452, q-alg/9509003.

[7] Lebedev N.N., Special functions and their applications, Dover Publications, Inc., New York, 1972.

[8] Ludwig D., The Radon transform on Euclidean space, Comm. Pure. App. Math. 23 (1966), 49-81.

[9] Nessibi M.M., Rachdi L.T., Trimèche K., Ranges and inversion formulas for spherical mean operator and its dual, J. Math. Anal. Appl. 196 (1995), 861-884.

[10] Rosenblum M., Generalized Hermite polynomials and the Bose-like oscillator calculus, in Nonselfadjoint Operators and Related Topics (Beer Sheva, 1992), Oper. Theory Adv. Appl., Vol. 73, Birkhäuser, Basel, 1994, 369-396, math.CA/9307224.

[11] Rösler M., Bessel-type signed hypergroups on $\mathbb{R}$, in Probability Measures on Groups and Related Structures, XI (Oberwolfach, 1994), Editors H. Heyer and A. Mukherjea, Oberwolfach, 1994, World Sci. Publ., River Edge, NJ, 1995, 292-304.

[12] Rösler M., Generalized Hermite polynomials and the heat equation for Dunkl operators, Comm. Math. Phys. 192 (1998), 519-542, q-alg/9703006.

[13] Samko S.G., Hypersingular integrals and their applications, Analytical Methods and Special Functions, Vol. 5, Taylor \& Francis, Ltd., London, 2002.

[14] Solmon D.C., Asymptotic formulas for the dual Radon transform and applications, Math. Z. 195 (1987), 321-343.

[15] Soltani F., Trimèche K., The Dunkl intertwining operator and its dual on $\mathbb{R}$ and applications, Preprint, Faculty of Sciences of Tunis, Tunisia, 2000.

[16] Stein E.M., Singular integrals and differentiability properties of functions, Princeton University Press, Princeton, 1970.

[17] Trimèche K., Transformation intégrale de Weyl et théorème de Paley-Wiener associés à un opérateur différentiel singulier sur (0, ), J. Math. Pures Appl. (9) 60 (1981), 51-98.

[18] Trimèche K., The Dunkl intertwining operator on spaces of functions and distributions and integral representation of its dual, Integral Transform. Spec. Funct. 12 (2001), 349-374.

[19] Trimèche K., Paley-Wiener theorems for the Dunkl transform and Dunkl translation operators, Integral Transform. Spec. Funct. 13 (2002), 17-38.

[20] Xu Y., An integral formula for generalized Gegenbauer polynomials and Jacobi polynomials, Adv. in Appl. Math. 29 (2002), 328-343. 